\title{
Serum Ang and CysC Levels in Essential Hypertension Complicated by Hypertensive Emergencies
}

\author{
Junqiang Teng ${ }^{1}$, Rongtu Lei $^{2}$ and Xinwen Huang ${ }^{3}$ \\ ${ }^{1}$ Department of Internal Medicine, Beishan Street Community Health Service Center Of Hangzhou, China \\ ${ }^{2}$ Department of Cardiovascular, Zhejiang Provincial People's Hospital, People's Hospital of Hangzhou Medical College, China \\ ${ }^{3}$ Department of Emergency, Hangzhou Hospital of Zhejiang Medical and Health Group, China
}

\begin{abstract}
Objective: To explore the value of serum angiotensin II (Ang II) and cystatin C (CysC) levels in predicting hypertension emergency in patients with essential hypertension.

Study Design: Experimental study.

Place and Duration of Study: Hangzhou Hospital of Zhejiang Medical and Health Group, from January 2018 to February 2020.

Methodology: One hundred and thirty seven patients with essential hypertension were divided into hypertension emergency group (Group A, 42 cases) and essential hypertension group without emergency (Group B, 95 cases). Ninety-five healthy subjects were selected as control group. Correlation between serum Ang and CysC and essential hypertension complicated with hypertension emergency was analysed. Diagnostic ability of Ang and CysC was analysed by ROC curve.

Results: Serum Ang and CysC levels in Group A and B were higher than those in control group (all p <0.001); serum Ang II and CysC levels in Group A were higher than those in Group B (both $\mathrm{p}<0.001$ ). Serum Ang II and CysC levels were positively correlated with essential hypertension complicated with hypertension emergency $(r=0.480, p<0.001 ; r=0.485, p<0.001$, respectively). Area under ROC curve of serum Ang II combined with CysC for predicting hypertension emergency was 0.905 (95\% Cl: 0.854-0.955), which was larger than that of serum Ang II, CysC alone.

Conclusion: Serum Ang II and CysC in patients with primary hyperemia complicated with hypertension emergency were higher. Combined detection of serum Ang II and CysC might be used as an effective reference index for predicting hypertension emergencies in patients with essential hypertension.
\end{abstract}

Key Words: Serum, Angiotensin II (Ang II), Cystatin C (CysC), Essential hypertension, Hypertension emergency.

How to cite this article: Teng J, Lei R, Huang X. Serum Ang and CysC Levels in Essential Hypertension Complicated by Hypertensive Emergencies. J Coll Physicians Surg Pak 2020; 30(05):467-470. DOI: https://doi.org/10.29271/jcpsp.2020.05.467.

\section{INTRODUCTION}

Hypertensive emergencies refer to a serious life-threatening clinical syndrome in which blood pressure suddenly and significantly rises (generally exceeding 180/120 mm Hg) in patients with essential and secondary hypertension under the action of some inducements, accompanied by acute damage to the functions of important target organs such as heart, brain and kidney. ${ }^{1}$

Hypertensive emergencies often cause serious dysfunction of target organs, such as acute heart failure with pulmonary edema, cerebral infarction, hypertensiveencephalopathy, cerebral hemorrhage or subarachnoid hemorrhage, unstable angina pectoris, acute myocardial infarction, sudden death coronary heart disease, eclampsia and aortic dissection, etc., which seriously endanger patients' lives. ${ }^{2}$

Correspondence to: Xinwen Huang, Department of Emergency, Hangzhou Hospital of Zhejiang Medical and Health

Group, 310012, China

E-mail: yvfhfa@163.com

Received: April 15, 2020; Revised: May 20, 2020;

Accepted: May 26, 2020

DOI: https://doi.org/10.29271/jcpsp.2020.05.467
In the treatment of emergency patients with essential hypertension, it is necessary to take prompt and effective measures at the first time to reduce blood pressure to a safe range and control organ failure at the same time, thus improving the prognosis of patients. ${ }^{3,4} T$ Therefore, it is of great clinical significance to find effective indicators to predict hypertension emergencies.

Studies have shown that under the action of various stress factors (such as severe mental trauma and excessive emotional excitement), sympathetic nerve tension and vasoconstrictive active substances in blood (such as angiotensin II, etc.) increase greatly, which can induce a sharp rise in blood pressure in a short period of time. ${ }^{5}$ Ang II cannot only promote vasoconstriction, but also promote the secretion of aldosterone (ALD). ALD is a very strong electrolyte regulator, which has the function of preserving sodium and excreting potassium, promoting the reabsorption of water and sodium at the same time, and then increasing blood volume and blood pressure. ${ }^{6}$ Angiotensin II (Ang II) has the greatest influence on the formation of left ventricular hypertrophy. ${ }^{7}$

Cystatin C (CysC) is a kind of low molecular weight protein that normally exists in serum and body fluids. It has a small molecular weight and positively charged. It can freely pass through the glomerular basement membrane and is reabsorbed and further metabolised in the proximal convoluted tubule. What's more, there is almost no renal tubule secretion, and the concentration in 
blood is also very constant, even under the influence of inflammatory state, it will not change, and will not be affected by various factors such as malignant tumor, inflammation, age, etc. ${ }^{8}$ Therefore, CysC can be used as an endogenous marker of glomerular filtration rate (GFR), as well as a clinically recognised effective indicator for diagnosing early renal injury with high sensitivity and specificity. ${ }^{9}$ At present, there are few reports on the application value of serum Ang II and CysC levels in predicting hypertension emergencies in patients with essential hypertension.

The objective of this study was to explore the value of serum angiotensin II (Ang II) and cystatin C (CysC) levels in predicting hypertension emergency in patients with essential hypertension.

\section{METHODOLOGY}

Approved by the Institutional Ethics Committee, this experimental study was conducted at Hangzhou Hospital of Zhejiang Medical and Health Group, China $\square$ from January 2018 to February 2020. One hundred and thirty-seven patients withessential hypertension were divided into hypertension emergency group (Group A, 42 cases) and essential hypertension group (Group B, 95 cases) based on the diagnosis result. Inclusion criteria were that patients in line with the diagnostic criteria for essential hypertension; patients who had not received antihypertensive medicines or statins for at least 2 weeks; patients with no previous hypertension emergency. Exclusion criteria were that patients with secondary hypertension, hyperlipidemia, diabetes and other diseases and autoimmune diseases; patients with severe cardiac function, renal insufficiency or acute bacterial infection with abnormal liver function; patients with mental illness or cognitive impairment. The diagnostic criteria for hypertension emergency were patients with abnormal increase of blood pressure combined with target organ damage, hypertensive encephalopathy, cardiac insufficiency, acute coronary syndrome, etc. Systolic blood pressure $>180 \mathrm{mmHg}$, diastolic blood pressure $>120$ $\mathrm{mmHg}(1 \mathrm{mmHg}=0.133 \mathrm{kPa})$; the onset time of the disease was within 1-2 hours, and the clinical manifestations mainly included headache, palpitation, dizziness, nausea, vomiting, chest pain and respiratory depression. At the same time, 95 healthy subjects with no obvious abnormality in various examination indexes were selected as healthy control (control group).

Table I: Comparison of serum Ang II and CysClevels.

\begin{tabular}{|l|c|c|c|c|}
\hline Parameter & $\begin{array}{c}\text { Group A } \\
(\mathbf{n = 4 2 )}\end{array}$ & $\begin{array}{c}\text { Group B } \\
(\mathbf{n = 9 5 )}\end{array}$ & $\begin{array}{c}\text { Control } \\
\mathbf{g r o u p} \\
(\mathbf{n = 9 5 )}\end{array}$ & p-value \\
\hline Ang II (pg/mL) & $\begin{array}{l}146.41 \\
\pm 10.56\end{array}$ & $\begin{array}{c}129.13 \\
\pm 6.50\end{array}$ & $87.47 \pm 3.95$ & $<0.001$ \\
\hline CysC (mg/L) & $1.53 \pm 0.22$ & $1.06 \pm 0.17$ & $0.83 \pm 0.06$ & $<0.001$ \\
\hline
\end{tabular}

Five $\mathrm{mL}$ of fasting elbow venous blood was drawn from all subjects when they were admitted to hospital, and the serum was centrifuged to separate for later use. The serum levels of Ang II and $\mathrm{Cys} C$ were detected by enzyme linked immunosorbent assay.

SPSS 25.0 software was used to analyse the collected data. The counting data were expressed as $n(\%)$. Kolmogrov-Smirnov test or Shapiro-Wilk test was used to evaluate of the normality of quantitative data. Measurement data with normal distribution were expressed as mean \pm SD. One-way ANOVA was used for multi-group comparison, and LSD-t test was used for further comparison. Spearman method was used to analyse the correlation between serum Ang II and CysC levels and essential hypertension complicated with hypertension emergency. The diagnostic ability of Ang II and CysC was analysed by receiver operating characteristic (ROC) curve respectively or in combination. The value $p$ $<0.05$ indicated that the difference was statistically significant.

\section{RESULTS}

Among the 137 patients with essential hypertension, 76 (55.47\%) were males and 61 (44.53\%) were females, aged $35-69$ years with an average of $52.81 \pm 2.65$ years.

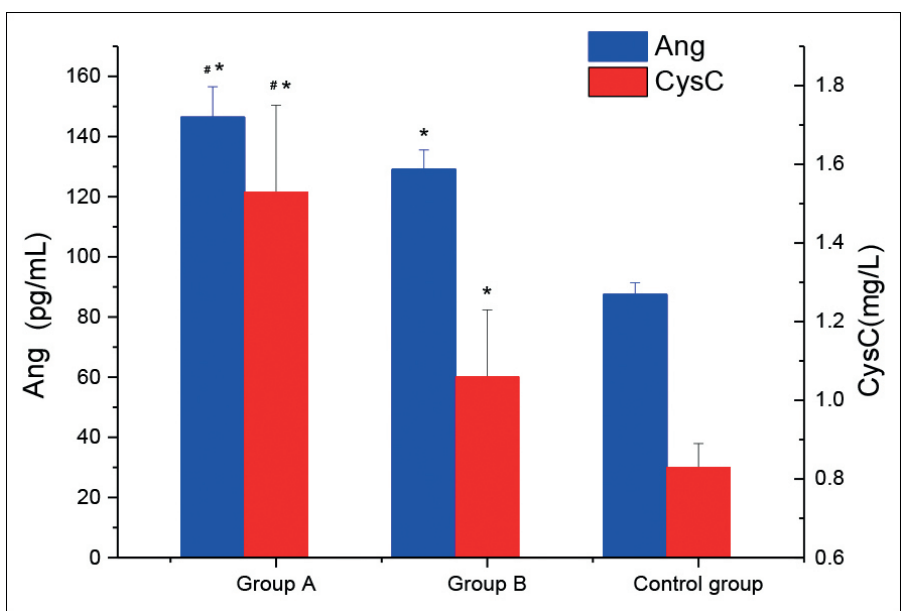

Figure 1: Comparison of serum Ang Il and CysC levels in three groups. $* \mathrm{P}<0.001$, vs control group; $\mathrm{p}<0.001$, vs Group $\mathrm{B}$.

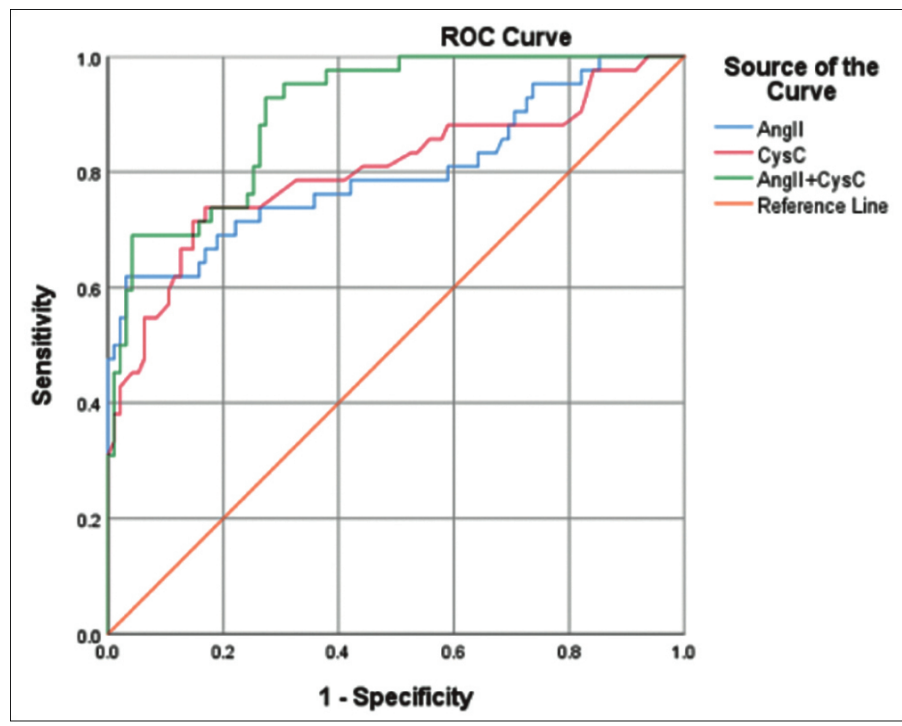

Figure 2: ROC curve of serum Ang II and CysC predicting essential hypertension complicated with hypertension emergency.

In Group A, there were 23 males (54.76\%) and 19 females (45.24\%), aged 36-69 years with an average of $53.75 \pm 2.81$ years. There were 8 cases $(19.05 \%)$ of hypertension complicated with acute left heart failure, 7 cases (16.67\%) of acute myocardial infarction, 10 cases $(23.81 \%$ ) of unstable angina pectoris, 9 cases (21.43\%) of hypertensive crisis, and 8 cases ( $19.05 \%)$ of hypertensive encephalopathy. In Group B, there were 53 males (55.79\%) and 42 females (44.21\%), aged 35-68 years, with an average of $52.06 \pm 2.37$ years. Among the 95 healthy subjects in the control group, 54 (56.84\%) were males and 41 (43.16\%) were females, aged 34-64 years with an average of $52.69 \pm 2.44$ years. 
The levels of serum Ang II and CysC in Group A and B were significantly higher than those in control group (all $p<0.001$, Table I and Figure 1); serum levels of Ang II and CysC in Group A were significantly higher than those in Group B (both $p<0.001$, Table I and Figure 1).

Spearman analysis showed that serum Ang II and CysC levels were positively correlated with essential hypertension complicated with hypertension emergency $(r=0.480, p<0.001$; $r=0.485, p<0.001$, respectively).

The analytical results of ROC curve showed that the area under ROC curve of serum Ang II combined with CysC for predicting hypertension emergency was 0.905 (95\% Cl:0.854-0.955), which was larger than that of serum Ang II [0.801(95\%Cl:0.709-0.893)] and CysC [0.804 (95\% $\mathrm{Cl}: 0.713-0.894)]$ for predicting hypertension emergency alone, as shown in Figure 2.

\section{DISCUSSION}

Ang II, the main active substance in renin angiotensin system, plays a very important role in the occurrence and development of cardiovascular diseases, such as stimulating the contraction of vascular smooth muscle cells to cause the increase of blood pressure, enhancing the retention of water and sodium by stimulating the secretion of aldosterone, and increasing hypertension by enhancing the excitability of sympathetic nervous system. ${ }^{10}$ Angiotensin is also a stimulating factor to promote the proliferation and hypertrophy of myocardial cells and vascular smooth muscle cells, and participates in the development of left ventricular hypertrophy and vascular remodelling in hypertension. ${ }^{11}$ The results of this study showed that the serum Ang II levels in patients with essential hypertension complicated with hypertension emergency and patients with essential hypertension were higher than those in healthy people. Ke et al. also found that the level of serum Ang II in patients with essential hypertension was higher than that in normal subjects. ${ }^{12}$

This study also found that serum Ang II level in patients with essential hypertension complicated with hypertension emergency was higher than that in patients with essential hypertension. This indicated that the serum Ang II level in patients with essential hypertension increased, and the emergency attack of hypertension would further promote the increase of Ang II level. It was suggested that serum Ang II might be a clinical diagnostic index forhypertension emergency.

Cys $\mathrm{C}$ is produced by nucleated cells and is a secretory inhibitor of cystine protease. It can be filtered through glomerulus and completely reabsorbed and degraded in renal tubules. Hypertensive renal damage is a long-term and continuous process with no obvious clinical symptoms in the early stage. ${ }^{13,14}$ When glomerulus is slightly damaged, CysC in blood can be increased.

Watanabe et al. confirmed that serum CysC was a useful and simple indicator to evaluate renal function in patients with essential hypertension, as well as an important early indicator to evaluate the severity ofterminal organ damage in patients with hypertension. ${ }^{15}$

Astudy hasfound that CysC is positively correlated with microalbuminuria as an early marker of renal damage and cardiovascular risk in patients with essential hypertension. ${ }^{16}$
The results of this study showed that the serum CysC level of patients with essential hypertension complicated with hypertension emergency and patients with essential hypertension was higher than that of healthy personnel, while the serum CysC level of patients with essential hypertension complicated with hypertension emergency was higher than that of patients with essential hypertension. It was suggested that serum CysC might become a potential diagnostic index for hypertension emergencies.

From simple essential hypertension to complicated hypertension emergency, it often indicates the further aggravation of the patient's condition. ${ }^{17}$ The results of this study showed that the levels of serum Ang II and CysC were positively correlated with essential hypertension complicated with hypertension emergency. The area under ROC curve, sensitivity and specificity of combined diagnosis of essential hypertension complicated with hypertension emergency were better than those of serum Ang II and CysC alone. It was suggested that serum Ang II and CysC levels could reflect the onset of hypertension emergencies and have high diagnostic efficiency for the presence or absence of hypertension emergencies.

\section{CONCLUSION}

The serum Ang II and CysC levels in patients with primary hyperemia complicated with hypertension emergency were significantly higher. Combined detection of serum Ang II and CysC levels in patients with essential hypertension had good predictive value for hypertension emergencies. Combined detection of serum Ang $\mathrm{II}$ and CysC might be used as an effective reference index for predicting hypertension emergencies in patients with essential hypertension.

\section{ETHICAL APPROVAL:}

This study has been approved by the Ethics Committee of Hangzhou Hospital of Zhejiang Medical and Health Group, China.

\section{PATIENTS' CONSENT:}

Informed consents were obtained from all participants.

\section{CONFLICT OF INTEREST:}

Authors declared no conflict of interest.

\section{AUTHORS' CONTRIBUTION:}

JT: Contributed to design articles, and authored the manuscript. RL: Revised the manuscript.

$\mathrm{XH}$ : Contributed to design articles, collected data, and approved the final manuscript.

\section{REFERENCES}

1. Saladini F, Mancusi C, Bertacchini F, Spannella F, Maloberti A, Giavarini $A$, et al. Diagnosis and treatment of hypertensive emergencies and urgencies among Italian emergency and intensive care departments. Results from an Italian survey: Progetto GEAR (Gestione dell'Emergenza e urgenza in ARea critica). Eur J Intern Med 2020; 71:50-6.

2. Sabir MS, Ali Khan MA, Hasan N. Hypertensive encephalopathy: A rare presentation of Williams-Beuren Syndrome. J Coll Physicians Surg Pak 2011; 21(8):509-10.

3. Van den Born BH, Lip GYH, Brguljan-Hitij J, Cremer A, Segura $J$, Morales E, et al. ESC Council on hypertension position document on the management of hypertensive emergencies. Eur Heart J Cardiovasc Pharmacother 2019; 5(1):37-46. 
4. Suneja M, Sanders ML. Hypertensive Emergency. Med Clin North Am 2017; 101(3):465-78.

5. Sayk F, Wobbe I, Twesten C, Meusel M, Wellhöner P, Derad I, et al. Prolonged blood pressure elevation following continuous infusion of angiotensin II-a baroreflex study in healthy humans. Am J Physiol Regul Integr Comp Physiol 2015; 309(11):R1406-14.

6. Fu Y, Ge S, Qiu X, Cui R, Zhang C, Xu X, et al. Effect of sample delivery conditions on renin-angiotensin-aldosterone system (RAAS) assay. Scand J Clin Lab Invest 2020; 1-7.

7. Alammari AH, Shoieb SM, Maayah ZH, El-Kadi AOS. Fluconazole represses cytochrome p450 $1 \mathrm{bl}$ and its associated arachidonic acid metabolites in the heart and protects against angiotensin ii-induced cardiac hypertrophy. J Pharm Sci 2020; S0022-3549(20)30175-1.

8. Amin F, Khan MS, Bano B. Mammalian cystatin and protagonists in brain diseases. J Biomol Struct Dyn 2020; 38(7):2171-96.

9. Sorkhi H, Behzadi R, Joghtaei N, Poornasrollah M, Bijani A. Glomerular filtration rate determination by creatinine and cystatin-C in patients with acute pyelonephritis. Caspian J Intern Med 2018; 9(3):290-5.

10. Urwyler SA, Ebrahimi F, Burkard T, Schuetz P, Poglitsch M, Mueller $B$, et al. IL (interleukin)-1 receptor antagonist increases ang (angiotensin [1-7]) and decreases blood pressure in obese individuals. Hypertension 2020; 75(6):1455-63.

11. Wang S, Li Y, Miao W, Zhao H, Zhang F, Liu N, et al. Angio- poietin-like protein 2 expression is suppressed by angiotensin II via the angiotensin II type 1 receptor in rat cardiomyocytes. Mol Med Rep 2016; 14(3):2607-13.

12. Ke YS, Tao YY, Yang H, Yu GH. Effects of valsartan with or without benazepril on blood pressure, angiotensin II, and endoxin in patients with essential hypertension. Acta pharmacol sin 2003; 24(4):337-41.

13. Hayakawa $Y$, Komaki H, Minatoguchi S, Yamada $Y$, Kanamori $\mathrm{H}$, Nishigaki $\mathrm{K}$, et al. High-salt intake accelerates functional and histological renal damage associated with renal tissue overexpression of (pro)renin receptors and AT1 receptors in spontaneously hypertensive rats. Clin Exp Nephrol 2020; doi: 10.1007/s10157-020-01888-7.

14. Tsai BC, Hsieh DJ, Lin WT, Tamilselvi S, Day CH, Ho TJ, et al. Functional potato bioactive peptide intensifies Nrf2-dependent antioxidant defense against renal damage in hypertensive rats. Food Res Int 2020; 129:108862.

15. Watanabe S, Okura T, Liu J, Miyoshi K, Fukuoka T, Hiwada K, et al. Serum cystatin $\mathrm{C}$ level is a marker of end-organ damage in patients with essential hypertension. Hypertens Res 2003; 26(11):895-9.

16. Moura Rdo S, Vasconcelos DF, Freitas E, Moura FJ, Rosa TT, Veiga JP. Cystatin C, CRP, log TG/HDLC and metabolic syndrome are associated with microalbuminuria in hypertension. Arq Bras Cardiol 2014; 102(1):54-9.

17. Tocci G, Presta V, Volpe M. Hypertensive crisis management in the emergency room: time to change? J Hypertens 2020; 38(1):33-4. 